\title{
Two cases of bilateral acute angle-closure glaucoma after radical surgery for cervical cancer with general anesthesia
}

\author{
Yue Li , Manhua Cui, Yingli Lu \\ Department of Gynecology and Obstetrics, Second Hospital of Jilin University, Chang Chun, Jilin 130041, China \\ Yue Li, E-mail: yueli_ju@163.com \\ Corresponding author, Manhua Cui :E-mail: cuimanhua@126.com \\ Yingli Lu: E-mail: luyl@jlu.edu.cn
}

\begin{abstract}
Cervical cancer is one of the common malignant tumours in gynecology. So far, its global occurrence rate is the highest among malignancies of female's reproductive system and the death rate is the second highest. Glaucoma is a blinding condition which is characterized by pathological intraocular hypertension, or damage of optic disc and retinal nerve fibre layer, and glaucomatous visual change under normal intraocular pressure. Typical clinical manifestations are sudden rise of intraocular pressure along with headache, nausea, vomiting, halo vision, eye distension and blurred vision. [1] Two main types of glaucoma are open-angle and angle-closure glaucoma. Primary acute angle-closure glaucoma is one of the emergencies in ophthalmology and is caused by the sudden rise of the intraocular pressure secondary to the difficulty of aqueous humor drainage from angle closure. Without timely treatments, the risk of blindness is extremely high. For the severe case, it can cause total blindness within a few days. Inappropriate treatments can also influence treatment effectiveness. [1] It is worthwhile to investigate the causes of these two concurrent diseases which are hardly related. In this article, we report two cases where acute angle-closure glaucoma developed after the radical surgery of cervical cancer with general anesthesia. We discuss the possible causes with respect to gene, anatomical and physiological factors, psychological factors, and preoperative narcotics, aiming to serve as clinical reference.
\end{abstract}

KEYWORDS: Glaucoma; Cervical cancer; General anesthesia

\section{INTRODUCTION}

A 55-year-old woman (gravida 1, parity 1; menopausal period 1 year) was diagnosed with Stage IIA1 cervical cancer. She had had excision of Bartholin gland cyst 21 years ago. She had no history of hypertension or glaucoma. Pathological diagnosis showed moderately differentiated squamous cell carcinoma of the uterine cervix. Human papillomavirus (HPV) value was 3000.00 (normal reference value < 1.00). TP protocol (paclitaxel $220 \mathrm{mg}+$ cisplatin $90 \mathrm{mg}$ ) was used to conduct radical hysterectomy, bilateral adnexectomy and pelvic lymphadenectomy after neoadjuvant chemotherapy. Two days after the surgery, the patient expressed headache and pain in both eyes. She was then diagnosed with acute angleclosure glaucoma. The suggested treatments were as follows: 1. Intravenous injection of $20 \%$ mannitol (50 mL); 2. alphagan (twice/day); 3. azopt (three times/day); 4. fuming pian (oral, 5 pills/time, three times/day); 5. pilocarpine nitrate eye drops (once/30 min); 6. TobraDex eyedrops (three times/day). Having treated with the above suggestions, the patient expressed significantly relieved headache and eye pains four days after the surgery. Postoperational pathology showed moderately differentiated squamous cell carcinoma of the uterine cervix with 1/2 layers of immersion into the cervix of the uterus wall, cancer thrombus in the vessel, and no cancer transfer in pelvic lymph nodes. 
The patient recovered well after the operation and concurrent chemoradiotherapy was recommended.

Another patient (56 years old; gravida 5, parity 3; menopausal period 7 year) was diagnosed with Stage IIA1 cervical cancer. She had had appendectomy 30 years ago and sterilization 26 years ago. She had no history of hypertension or glaucoma. Pathological diagnosis showed moderately differentiated squamous cell carcinoma of the uterine cervix. Human papillomavirus (HPV) value was 1000.30 (normal reference value $<1.00$ ). TP protocol (paclitaxel $240 \mathrm{mg}+$ cisplatin $90 \mathrm{mg}$ ) was used to conduct radical hysterectomy, bilateral adnexectomy and pelvic lymphadenectomy after neoadjuvant chemotherapy. Three days after the operation, the patient expressed eye discomfort and blurred vision. She was then diagnosed with acute angle-closure glaucoma. The suggested treatments were as follows: 1. Intravenous injection of mannitol (once or twice/day); 2. pilocarpine nitrate eye drops (once/5 min, three times in total; once/10 min, three times in total; once/15 min, three times in total); 3 . methazolamide (one drop/time for both eyes); 4 . alphagan (one drop/time for both eyes). Having treated with the above suggestions, the patient expressed significantly relieved eye discomfort seven days after the surgery. Postoperational pathology showed moderately differentiated squamous cell carcinoma of the uterine cervix with minor immersion into the cervix of the uterus wall, no confirmed immersion in the vessel with vaginal wall, no cancer in the vaginal cuff (tumor of some parts near the rim), no cancer in bilateral adnexa, atrophic endometrium, and no cancer transfer in pelvic lymph node. The patient recovered well after the operation and concurrent chemoradiotherapy was recommended.

\section{Discussion}

\subsection{From gene level}

Since the first Forkhead gene of the FOX (forkhead box) transcription factor family was cloned in 1989, over 100 members have been identified so far. All FOX family members share a highly conserved DNA-binding domain with 110 amino acids, which is also called the forkhead box. [2] All FOX proteins can bind DNA through the unique DNA-binding domain. The forkhead genes influence the maintenance of cell differentiation, the expressions of tissuespecific genes, embryonic development, cellcycle control, carbohydrate and lipid metabolism, apoptosis and other biological processes. [3] Due to the unique sequence of the DNA-binding domain and the different mechanisms of molecular interactions with target DNAs for different FOX proteins, their functions vary from activation to inhibition of genetic transcription. Based on the important roles of FOX proteins in various fundamental developments and tissue autoregulation processes, the deficiency or acquisition of FOX protein functions can change cell fate and even lead to tumorigenesis. [4] Among the various FOX proteins, Foxc1, Foxc2, Foxd1, Foxe 3 and Foxg1 are related to eye development, so their mutations will cause glaucoma, goniosynechia and iris dysplasia. [5] Meanwhile, FOXM1 gene expression level is seven-fold above normal in primary HPV16- and HPV18-infected early stage cervical cancers. The overexpression of FOXM1 may cause HPV-induced keratinocyte transformation and cervical cancer. [6] Therefore, we can speculate that the possible cause of glaucoma and cervical cancer in the reported two cases is related to the abnormality of FOX genes. Even though neither patient had glaucoma history, they may possibly have been at high risk of glaucoma due to the abnormal genes. The abnormality of FOX genes can certainly establish a connection between the two rarely-related diseases and become the possible cause of the two diseases. 


\subsection{Anatomical and physiological factors}

Primary angle-closure glaucoma is the most common type of glaucoma among Chinese population. Its occurrence rate is especially high for females over 50 years old. The two patients reported here are 55 and 56 years old, respectively. With age, the thickness of the lens increases along with shallower anterior chamber and increased pupil blockage. Once the surrounding iris contacts the trabecular network, the chamber angle then closes and the intraocular pressure increases to trigger an acute attack of glaucoma. [7] Moreover, elderly people often have exfoliated iris pigment cells and lens epithelial cells, which flow with the aqueous humor and can be suspended on the trabecular network when flowing through it. The flexibility of the trabecular network deteriorates with age, which has negative effects on its filtering function. Apart from age, anatomic abnormalities, such as congenital shallow anterior chamber, short axis depth and small chamber angle, cannot be excluded and can influence the reflow of aqueous humor. [7] The above factors can possibly become the basis of potential high intraocular pressure and trigger acute angle-closure glaucoma.

\subsection{Psychological factors}

Nervousness before surgery, ineffective anesthesia, trauma and pain during operation, and postoperative pain can lead to enhanced excitability of the sympathetic nervous system and release of adrenalin or similar. These further lead to dilated pupil to make surrounding iris and trabecular network contact each other so that the discharge of aqueous humor is blocked to increase intraocular pressure and induce an acute attack of angle-closure glaucoma.

\subsection{Preoperative narcotics}

From the anesthesia records, it was found that atropine and succinylcholine were used for both patients. Atropine is an anticholinergic agent. It is intramuscularly injected to reduce the bronchial mucus secretion during anesthesia to prevent respiratory obstruction and aspiration pneumonia. However, atropine also has the effect of dilating pupil and increasing intraocular pressure so as to induce glaucoma. Succinylcholine, which is often used during anesthetic induction, is the synthetic substitute for tubocurarine. Fast muscle relaxation, short duration and easy control make succinylcholine suitable for surgery where it makes tracheal cannula easy to perform. However, succinylcholine can also lead to the contraction of extraocular muscles and the forward shift of crystal iris diaphragm so that the anterior chamber becomes shallower to increase intraocular pressure, resulting in acute angleclosure glaucoma. [8] Therefore, much attention should be paid before operations especially for middle-aged and elderly female patients. Consultations with the ophthalmologists are necessary for patients who will be or have been administered with atropine and succinylcholine during the operation so as to shorten the duration of acute ocular hypertension and reduce vision damage to the minimum. Doctors should pay adequate attention to the fact that the induced acute attack of glaucoma is often concealed by the pain from the operation. [9] Meanwhile, anesthesiologists should enhance their recognition of angle-closure glaucoma so that its acute attack from the use of atropine or similar can be dealt with timely during the perioperative period.

\subsection{Prevention and treatment methods}

Glaucoma is an eye disease which is mainly characterized by increased intraocular pressure, along with typical decreased visual function and ocular tissue damage. If treatment is delayed, it can easily cause blindness. So active preventive measures should be taken. Ophthalmic examinations should be performed before surgery, including queries of past eye diseases and exclusion of shallow anterior chamber. Patient should be comforted to ease the psychological tension before and after 
operations. For elderly patients, attention should be paid to the likely occurrence of acute glaucoma by applying medications to reduce intraocular pressure for part or whole body during and after surgeries. Study [10] suggests that dehydration along with lasting miosis during surgeries can treat acute angle-closure glaucoma induced by the use of atropine for general anesthesia. The patient's risk of angleclosure glaucoma should be assessed before surgery. It is recommended to apply $2 \%$ pilocarpine nitrate for miosis before surgery, and pilocarpine during surgery supplemented by timolol eye drops and alphagan ointment (once/10 min) until the end of the operation and anesthesia recovery. As long as there is acute attack of glaucoma during or after surgery, active measures to decrease intraocular pressure should be taken to prevent impairing vision due to long-term high intraocular pressure. Close observations should be conducted as well after the surgery. If any patient expresses eye pain, blurred vision or bloodshot eyes, great attention should be paid to the likelihood of acute angleclosure glaucoma. Early discovery, prevention and timely treatments can prevent vision damage.

\section{CONCLUSIONS}

In conclusion, great attentions and vigilance from anesthesiologists and clinicians should be aroused for the occurrence of such situations described herein. Great efforts should be made to reduce the occurrence rate of complications after operations. Make sure to prevent, discover, diagnose and treat such diseases early to earn excellent prognosis for patients.

\section{References}

[1] Zhou W. Clinical glaucoma. Beijing: People's Medical Publishing House. 2000: 171. Chinese.

[2] Kaestner KH, Knochel W, Martinez DE. Unified nomenclature for the winged helix/forkhead transcription factors. Genes Dev. 2000, 14(2): 142-146.

[3] Lehmann OJ, Sowden JC, Carlsson P, Jordan T, Bhattacharya SS. Fox's in development and disease. Trends Genet. 2003, 19(6): 339-344.

[4] Kaufmann E, Knöchel W. Five years on the wings of fork head. Mech Dev. 1996, 57(1): 320 .

[5] Carlsson P, Mahlapuu M. Forkhead transcription factors: key players in development and metabolism. Dev Biol. 2002, 250(1): 1-23.

[6] Santin AD, Zhan F, Bignotti E, et al. Gene expression profiles of primary HPV16- and HPV18-infected early stage cervical cancers and normal cervical epithelium: identification of novel candidate molecular markers for cervical cancer diagnosis and therapy. Virology. 2005, 331(2): 269-291.

[7] Zhao K, Yang P. Ophthalmology. Beijing: People's Medical Publishing House. 2013: 167. Chinese.

[8] Guo Y. One case of acute angle-closure glaucoma induced by general anesthesia. Chinese Journal of Ophthalmology and Otorhinolaryngology. 2005, 5(5): 321.

[9] Geng M, Yin Y, Zhai J. Analysis of acute angle-closure glaucoma induced by atropine in general anesthesia. Medical Journal of National Defending Forces in Southwest China. 2008, 18(1): 26 .

[10] Wu L, G M. Dehydration combined with continuous intraoperative pupil dilation for acute angle-closure glaucoma caused by misusing of atropine in anesthesia. Anhui Medical Journal. 2014, 35(6): 826-828. 